УДК 697.12

\title{
РАСЧЕТ КОЛИЧЕСТВА ПОТРЕБЛЯЕМОЙ ТЕПЛОВОЙ ЭНЕРГИИ ПРИ ОБОГРЕВЕ ПОМЕЩЕНИЯ ЧУГУННЫМ РАДИАТОРОМ М-140. ЧАСТЬ 1. СТАЦИОНАРНЫЙ РЕЖИМ ОТОПЛЕНИЯ
}

Басок Б.И., член-корреспондент НАН Украины, Давыденко Б.В., докт. техн. наук, Тимощенко А.В., канд. техн. наук

Институт технической теплофизики НАН Украинь,, ул. Желябова, 2а, г. Киев, 03б80, Украина

3 використанням наближеної теплофізичної моделі теплопереносу в приміщенні, що опалюється чакількість теплової енергії, необхідної для підтримки в приміщенні нормативної для зимового періоду температури.
С использованием приближенной теплофизической модели теплопереноса в помещении, отапливаемом чугунным радиатором, энергии, необходимой для поддержа- reg ния в помещении нормативной для room. зимнего периода температуры.

\section{Библ. 3, рис. 2.}

Ключевые слова: обогрев помещения, температурный режим, радиатор.

$c_{0}, \mathrm{BT} /\left(\mathrm{M}^{2} \mathrm{~K}^{4}\right)$ - коэффициент излучения абсолют но черного тела;

$F$, м $^{2}$ - площадь одной секции радиатора;

$G, \mathrm{M}^{3} /$ час - расход;

m, кГ - масса;

$n$ - количество секций радиатора;

$Q$, Вт - тепловой поток;

$R, \mathrm{M}^{2} \mathrm{~K} / \mathrm{B} \mathrm{T}-$ термическое сопротивление;

$\mathrm{t},{ }^{0} \mathrm{C}$ - температура;

$\alpha, \mathrm{BT} /\left(\mathrm{M}^{2} \mathrm{~K}\right)$ - коэффициент теплоотдачи;

$\varepsilon-$ степень черноты;

$\lambda, \mathrm{BT} /(\mathbf{M} \cdot \mathrm{K})$-коэффициент теплопроводности;

\section{Введение}

В связи с повышением стоимости коммунальных услуг в Украине, у населения часто возникают вопросы относительно обоснованности установленного тарифа на отопление их квартир. На сегодняшний день в жилом секторе начисление суммы, которую необходимо оплатить за отопление, производится по двум возможным схемам в зависимости от наличия или отсутствия специальных устройств для учета потребленной тепловой энергии. В случае наличия такого устройства устанавливается тариф за единицу потребленной энергии, и оплата производит- $\varphi$ - коэффициент взаимного облучения поверхностей радиатора и поверхностей стен.

\section{Нижние индексы:}

в - воздух внутри помещения;

инф - инфильтрация;

к - конвективный;

л - лучистый;

н - наружный воздух;

о - окно;

p - радиатор;

с - стена.

т - теплоноситель.

ся в зависимости от количества этой энергии, затраченной на отопление за месяц. Однако в этом случае жильцы квартиры не всегда имеют возможность самостоятельно регулировать необходимое количество энергии на отопление. Это обусловлено тем, что в основном в жилых зданиях установлена однотрубная система отопления с последовательным подключением отопительных устройств. Установка устройств для регулирования температуры в системе отопления (при наличии технической возможности), а также принятие мер для уменьшения потерь теплоты через наружные ограждения помещений, 
позволит организовать оптимальный температурный режим в помещениях и в зависимости от этого отрегулировать приемлемую для жильцов оплату за отопление. Недостатком такой схемы оплаты является достаточно сложная процедура получения разрешения на установку счетчиков и высокая стоимость самих счетчиков.

По другой схеме, если фиксация потребленной теплоты не проводится, оплата начисляется в соответствии с размером отапливаемой площади квартиры. Сумма начисления в этом случае не зависит от мер по утеплению помещения, а также от качества предоставляемой коммунальной услуги, то есть независимо от того, тепло ли в квартире, или холодно.

Тарифы на потребленную теплоту, как в случае первой, так и второй схемы оплаты, различны для различных областей Украины. Они зависят от технического состояния тепловых сетей, производительности котельных, от вида и сорта топлива и его цены, климатических условий и ряда других факторов. Но в любом случае должно соблюдаться определенное соответствие между тарифом, рассчитанным по объему реально потребленной тепловой энергии, и тарифом, что начисляется, исходя из отапливаемой площади помещения.

\section{Постановка задачи}

При отсутствии в помещении средств для фиксации потребляемой тепловой энергии, ее приближенно можно оценить расчетным путем. Если, например, отопление осуществляется с помощью чугунных секционных радиаторов, то с их поверхностей в помещение поступает конвективный тепловой поток $Q_{\text {к }}$ и лучистый тепловой поток $Q_{\text {л }}$. Теплообмен естественной конвекцией происходит между поверхностью радиатора с температурой $t_{\mathrm{p}}$ и воздухом в помещении с температурой $t_{\text {в }}$ Радиационный теплообмен происходит между поверхностью радиатора и поверхностями стен помещения, имеющих температуру $t_{c}$. Суммарный тепловой поток от радиатора при этом равен

$$
Q_{\mathrm{p}}=Q_{\mathrm{\kappa}}+Q_{\text {л }}
$$

Используя для расчета коэффициента теплоотдачи естественной конвекцией формулу

$$
\alpha_{\mathrm{\kappa}}=1,66\left(t_{\mathrm{p}}-t_{\mathrm{B}}\right)^{1 / 3},
$$

представленную в [1], можно получить выражение для лучисто-конвективного теплового потока от $n$ секций радиатора

$$
\begin{aligned}
& \mathrm{Q}_{\mathrm{p}}=n F\left\{1,66 \cdot\left(t_{\mathrm{p}}-t_{\mathrm{c}}\right)^{4 / 3}+\right. \\
& \left.+\frac{\varphi \cdot c_{0}}{1 / \varepsilon_{\mathrm{p}}+1 / \varepsilon_{\mathrm{c}}-1}\left[\left(\frac{t_{\mathrm{p}}+273}{100}\right)^{4}-\left(\frac{t_{\mathrm{c}}+273}{100}\right)^{4}\right]\right\} .
\end{aligned}
$$

Для расчета суммарного теплового потока от чугунного радиатора М-140 в [1] также предложена приближенная зависимость

$$
Q_{\mathrm{p}}=n F \cdot 650 \cdot\left(\frac{t_{\mathrm{p}}-t_{\mathrm{s}}}{70}\right)^{1,3},
$$

для которой принимается, что температура воздуха в помещении и температура внутренних поверхностей стен - одинаковы, то есть $t_{\text {c }}=$ $t_{\text {в }}$ Значение $Q_{\mathrm{p}}$, рассчитанное по формуле (1) для $t_{\mathrm{B}}=20{ }^{\circ} \mathrm{C}$ при $40{ }^{\circ} \mathrm{C}<t_{\mathrm{p}}<65{ }^{\circ} \mathrm{C}$, превосходит значение $Q_{\mathrm{p}}$, рассчитанное по формуле (2), на $5.7 \%$. Причем различие указанных значений уменьшается с увеличением $t_{\mathrm{p}}$.

Следует отметить, что рассчитать количество энергии для отопления помещения непосредственно по формулам (1) и (2) при априорном

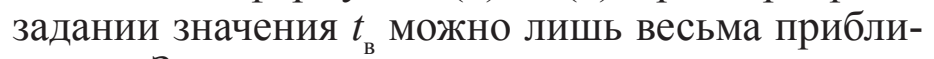
женно. Значения температуры воздуха в помещении $t_{\text {в }}$ входящие в формулы (1) и (2), сами также зависят как от температуры радиатора $t_{\mathrm{p}}$, так и от температуры наружного воздуха $t_{\text {н }}$, а также от термического сопротивления ограждающих конструкций. Более точный результат может быть получен при расчете процесса переноса теплоты от радиатора к внешнему воздушному пространству через воздушную среду помещения и ограждающие конструкции. Данной расчет может быть произведен с использованием приближенной методики, основанной на теплофизической модели помещения с сосредоточенными параметрами [2]. Эта методика заключается в том, что температурные поля отдельных элементов теплофизической системы заменяются дискретными значениями их среднеинтегральной температуры. Вместо трехмерных элементов системы рассматриваются отдельные узлы, в которых сосредотачиваются геометрические 
и теплофизические характеристики указанных элементов. Для узлов составляются уравнения теплового баланса, которые учитывают тепловое взаимодействие между различными элементами системы.

Применительно к однокомнатному помещению принимается следующая модель теплопереноса от отопительного прибора к его элементам. Радиационный тепловой поток от части поверхности радиатора, обращенной вовнутрь помещения, поступает к внутренним стенам помещения, а также к полу и потолку. От части поверхности радиатора, обращенной к внутренней стене, радиационный поток поступает на ограниченный участок наружной стены, расположенный непосредственно за радиатором.

Радиационный тепловой поток между боковыми поверхностями соседних секций радиатора считается равным нулю. В связи с этим можно предположить, что лишь половина поверхности секции радиатора участвует в лучистом теплообмене со стенами помещения. Со всей поверхности радиатора теплота естественной конвекцией передается воздушной среде внутри помещения. От внутренних стен, пола и потолка радиационный тепловой поток поступает на поверхность наружной стены и на окно. Все внутренние поверхности помещения участвуют также в конвективном теплообмене с воздушной средой внутри помещения.

На температуру воздуха внутри помещения влияет также воздушный поток, который поступает в помещение из внешнего пространства за счет инфильтрации. От внешней поверхности наружной стены и от внешней поверхности окна теплота конвекцией передается во внешнюю среду. Предполагается, что через внутренние стены, пол и потолок теплота в соседние помещения не передается. Разница между тепловой энергией, подводимой к ограждениям, и энергией, отводимой от них, составляет количество тепловой энергии, которая аккумулируется данными элементами. За счет этого происходит изменение во времени среднеобъемной температуры указанных элементов. Система уравнений теплового баланса для отдельных элементов помещения, соответствующая указанной модели теплопереноса, приведена в [2].

\section{Анализ результатов расчетных исследования}

Рассмотренную теплофизическую модель можно использовать для определения температурного режима и количества потребляемой энергии для отопления однокомнатного помещения длиной 5 м; шириной 3 м и высотой 3 м (отапливаемая площадь $15 \mathrm{~m}^{2}$ ). Внешняя и внутренняя стены толщиной 0,3 м выполнены из кирпича $(\lambda=0,56 \mathrm{BT} /(\mathrm{M} \cdot \mathrm{K}))$. Площадь окна составляет $2,5 \mathrm{~m}^{2}$. Его термическое сопротивление (без учета сопротивлений внутренней и внешней теплоотдачи) составляет $R=0,18 \mathrm{M}^{2} \cdot \mathrm{K} / \mathrm{BT}$. Степень черноты всех поверхностей теплообмена принимается равной $\varepsilon=0,85$. Принимается также, что кратность воздухообмена в помещении за счет нерегулируемой вентиляции и инфильтрации наружного воздуха составляет $0,4\left(G_{\text {инф }}=18 \mathrm{~m}^{3} /\right.$ час). Коэффициент теплоотдачи к наружному воздуху $-\alpha_{\mathrm{H}}=23,0 \mathrm{BT} /\left(\mathrm{M}^{2} \mathrm{~K}\right)$.

В качестве отопительного устройства используется чугунный радиатор М-140 с количеством секций $n=10$. Масса одной секции чугунного радиатора $m_{\mathrm{p}}=6,2$ кг, а ее поверхность $F=0,244$ м $^{2}$. Теплоносителем в системе отопления является вода, общая масса которой составляет $m_{\mathrm{T}}=14,5$ кг. Радиатор располагается около наружной стены помещения под окном.

Расчет стационарных температурных режимов данного помещения выполняется для различных значений температуры теплоносителя $t_{\mathrm{T}}$ на входе в отопительный прибор при трех значениях температуры наружного воздуха: $t_{\mathrm{H}}=0{ }^{\circ} \mathrm{C} ; t_{\mathrm{H}}=-5^{\circ} \mathrm{C} ; t_{\mathrm{H}}=-10^{\circ} \mathrm{C}$. Расход теплоносителя через радиатор составляет $0,1 \mathrm{~m}^{3} /$ час. Температура радиатора $t_{\mathrm{p}}$ принимается равной среднему значению температуры теплоносителя на входе и выходе из радиатора. Зависимости температуры воздуха в помещении $t_{\text {в }}$ и теплового потока $Q_{\mathrm{p}}$, поступающего от радиатора, от значений $t_{\mathrm{T}}$ представлены на рис. 1. Из рис. 1 видно, что при указанных выше условиях нормативная температура воздуха в помещении $t_{\mathrm{B}}=20^{\circ} \mathrm{C}$ обеспечивается при $t_{\mathrm{T}}:=48,05{ }^{\circ} \mathrm{C}$ для $t_{\mathrm{H}}=0{ }^{\circ} \mathrm{C}$; при $t_{\mathrm{T}}=53,67{ }^{\circ} \mathrm{C}$ для $t_{\mathrm{H}}=-5^{\circ} \mathrm{C}$ и при $t_{\mathrm{T}}=59,12{ }^{\circ} \mathrm{C}$ для $t_{\mathrm{H}}^{\mathrm{T}}=-10{ }^{\circ} \mathrm{C}$. Значения температуры радиатора составляют при этом $t_{\mathrm{p}}=46,14{ }^{\circ} \mathrm{C} ; t_{\mathrm{p}}=51,26{ }^{\circ} \mathrm{C}$ и $t_{\mathrm{p}}=56,21{ }^{\circ} \mathrm{C}$ соответственно. Этим условиям 
соответствуют значения теплового потока от радиатора $Q_{\mathrm{p}}=445,09$ Вт; $Q_{\mathrm{p}}=559,72$ Вт и $Q_{\mathrm{p}}=675,68$ Вт соответственно.

Из представленных результатов следует, что если в течение 30 дней температура наружного воздуха будет $t_{\mathrm{H}}=0{ }^{\circ} \mathrm{C}$, то для поддержания температуры в данном помещении на нормативном уровне $t_{\text {в }}=20{ }^{\circ} \mathrm{C}$, за указанный период будет израсходована тепловая энергия 1,15 ГДж/ мес $(0,275$ ГКал/мес). Если же температура наружного воздуха будет $t_{\mathrm{H}}=-10{ }^{\circ} \mathrm{C}$, то указанная величина составит 1,75 ГДж/мес (0,418 ГКал/мес). Отметим, что данные результаты получены для стационарных условий, при которых $t_{\mathrm{H}}$ и $t_{\mathrm{T}}$ не изменяются во времени.

Из анализа отдельных составляющих общих потерь теплоты рассматриваемого помещения следует, что при данных условиях 42 \% тепловой энергии переносится во внешнюю среду через поверхность наружной стены. Из них 7 \% передается через участок, расположенный непосредственно за радиатором (его площадь составляет $0,61 \mathrm{M}^{2}$ ), а $35 \%$ - через остальные участки поверхности стены. Тепловые потери через окно составляют 31 \%, а потери за счет инфиль-

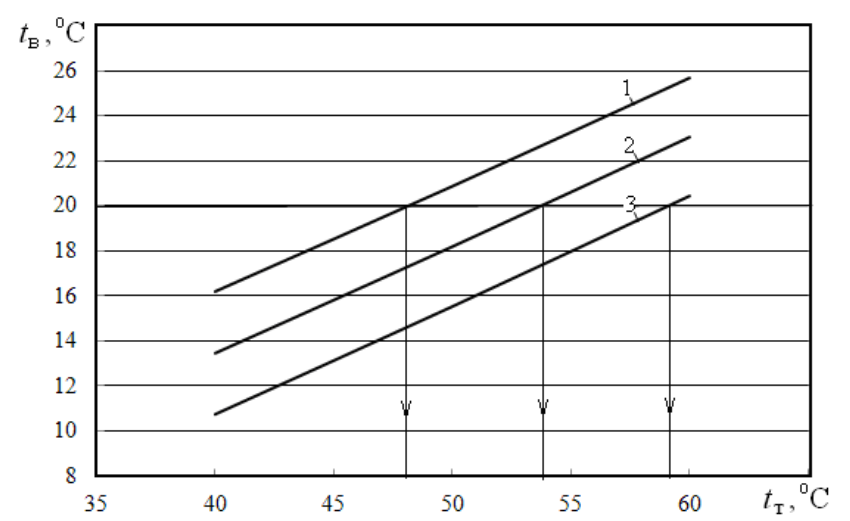

a) трации - 27 \% от суммарных тепловых потерь. Плотность теплового потока, направленного во внешнюю среду, на участке за радиатором в 1,8..1,9 раз выше, чем плотность теплового потока вне этого участка, что является следствием его значительного перегрева по сравнению с другими участками наружной стены. Перегрев участка стены за радиатором происходит за счет лучистого теплового потока, исходящего от радиатора. Для рассматриваемых диапазонов изменения температуры теплоносителя и температуры наружного воздуха, температура стены за радиатором оказывается на 7,9 ${ }^{\circ} \mathrm{C} \ldots 13,1{ }^{\circ} \mathrm{C}$ выше температуры воздуха внутри помещения. Температура же других участков наружной стены - на $3,7^{\circ} \mathrm{C} \ldots 6,4{ }^{\circ} \mathrm{C}$ ниже температуры воздуха в помещении. В этих условиях теплота с поверхности стены за радиатором переносятся теплопроводностью через стену во внешнюю среду, а также передается конвекцией воздушной среде внутри помещения, способствуя ее нагреву.При этом тепловой поток, направленный в сторону внешней среды в 1,4...1,6 раз выше, чем тепловой поток, передаваемый воздуху в помещении.

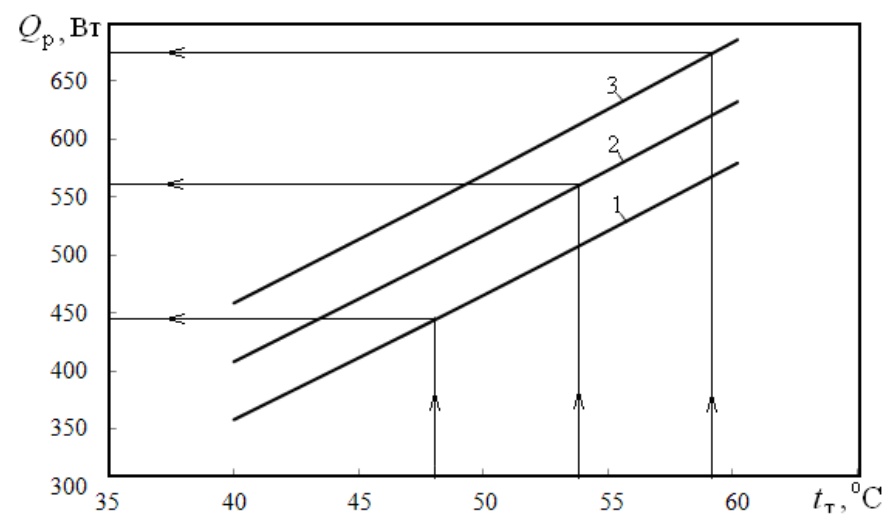

б)

Рис. 1. Зависимости температуры воздуха в помещении (а) и теплового потока (б), поступающего от радиатора, от температуры теплоносителя на входе в отопительный прибор: $1-t_{u}=0^{\circ} \mathrm{C} ; 2-t_{u}=-5^{\circ} \mathrm{C} ; 3-t_{u}=-10^{\circ} \mathrm{C}$.

Одним из рекомендованных способов снижения тепловых потерь через “зарадиаторный” участок стены считается установка на его поверхности теплоотражающего экрана со значительно меньшим коэффициентом теплового излучения, чем у поверхности самой стены. Для установления степени влияния теплоотражающего экрана на общие потери теплоты через стену, задача теплопереноса в помещении в рассмотренной выше постановке решается при условии, что 
степень черноты участка поверхности стены за радиатором составляет $\varepsilon=0,09$. Коэффициент черноты других участков стены $-\varepsilon=0,85$. Зависимости температуры воздуха в помещении $t_{\text {в }}$ и теплового потока $Q_{\mathrm{p}}$, поступающего от радиатора, от температуры теплоносителя $t_{\mathrm{H}}$ на входе в радиатор для данного случая представлены на рис. 2. Пунктиром на данном рисунке представлены зависимости, соответствующие рассмотренному выше случаю, когда степень черноты всей поверхности наружной стены одинакова и равна $\varepsilon=0,85$. Как видно из рис. $2, \sigma$, тепловые потоки от радиатора, а также и равные им суммарные тепловые потери, в случае установки за радиатором теплоотражающего экрана снижаются на 6,1 \%...6,5 \% по сравнению со случаем

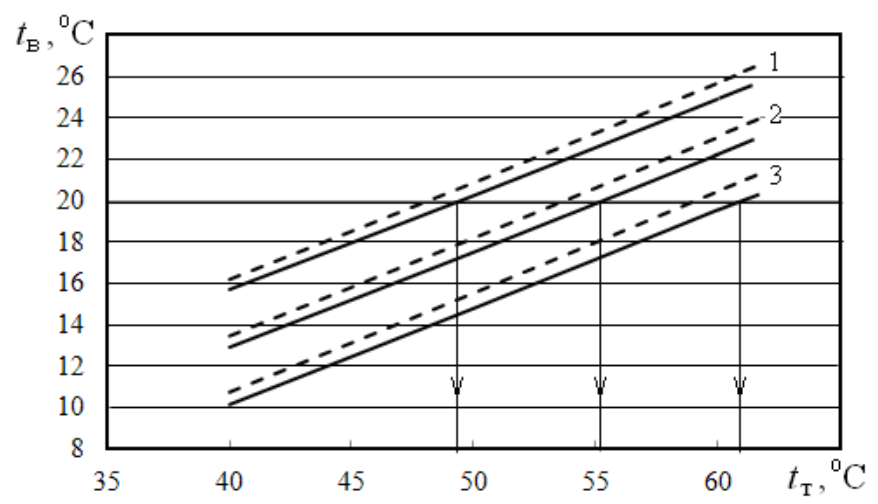

a) отсутствия экрана. При этом тепловые потоки во внешнюю среду через участок стены за радиатором уменьшаются в $1,8 \ldots 1,9$ раза. Вместе с тем, как видно из рис. 2,a, при одних и тех же значениях температуры теплоносителя на входе в отопительный прибор, температура воздуха в помещении при наличии теплоотражающего экрана снижается на $0,5 \ldots 1,0{ }^{\circ} \mathrm{C}$ по сравнению со случаем отсутствия экрана. Это является следствием значительного уменьшения температуры стены за радиатором. При наличии теплоотражающего экрана температура стены за радиатором становится на $3,3 \ldots .5,6^{\circ} \mathrm{C}$ ниже температуры воздуха в помещении, и тепловой поток в этом случае передается конвекцией от воздуха к стене, как и на других участках наружной стены.

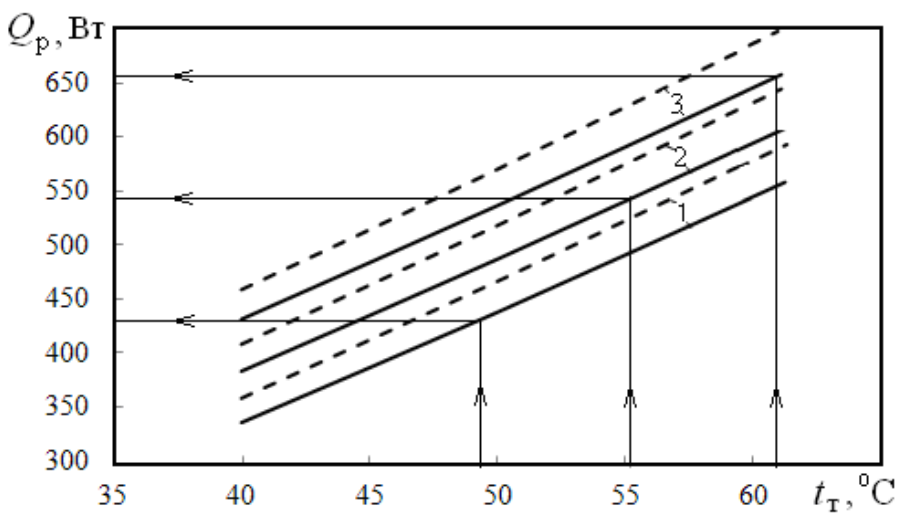

б)

Рис. 2. Зависимости температуры воздуха в помещении (а) и теплового потока (б), поступающего от радиатора, от температуры теплоносителя на входе в отопительный прибор при наличии теплоотражающего экрана (сплочная линия) и при его отсутствии (пунктир):

$$
1-t_{u}=0^{\circ} \mathrm{C} ; 2-t_{u}=-5^{\circ} \mathrm{C} ; 3-t_{u}=-10^{\circ} \mathrm{C} \text {. }
$$

В данных условиях для поддержания температуры в помещении на регламентированном уровне $t_{\mathrm{B}}=20{ }^{\circ} \mathrm{C}$ необходимо повысить температуру теплоносителя на входе в отопительный прибор. Из рис. 2, а видно, что значения температуры теплоносителя должны при этом составлять $t_{\mathrm{T}}=49,36{ }^{\circ} \mathrm{C}$ при $t_{\mathrm{H}}=0{ }^{\circ} \mathrm{C} ; t_{\mathrm{T}}=55,25{ }^{\circ} \mathrm{C}$ при $t_{\mathrm{H}}=-5{ }^{\circ} \mathrm{C}$ и $t_{\mathrm{T}}=60,98{ }^{\mathrm{H}} \mathrm{C}$ при $t_{\mathrm{r}}=-10^{\circ} \mathrm{C}$. Значения температуры радиатора составляют при этом $t_{\mathrm{p}}=47,5{ }^{\circ} \mathrm{C} ; t_{\mathrm{p}}=52,91{ }^{\circ} \mathrm{C}$ и $t_{\mathrm{p}}=58,15^{\circ} \mathrm{C}$ соответственно. Этим условиям соответствуют значения теплового потока от радиатора
$Q_{\mathrm{p}}=431,3$ Вт; $Q_{\mathrm{p}}=543,37$ Вт и $Q_{\mathrm{p}}=656,96$ Вт соответственно. Указанные величины теплового потока от радиатора, необходимые для поддержания в помещении температуры воздуха на уровне $t_{\text {в }}=20{ }^{\circ} \mathrm{C}$, оказываются на $3,1 \% \ldots 2,8 \%$ ниже значений, полученных ранее для случая отсутствия теплоотражающего экрана.

Представленные результаты относятся к системе отопления, в которой используется достаточно старая модель отопительного устройства - чугунный радиатор М-140. В настоящее время существует немало новых конструкций и марок 
отопительных устройств, которые считаются более эффективными с теплотехнической и экономической точек зрения, чем чугунные радиаторы М-140. Следует отметить, что, как правило, производители современных радиаторов в официальных паспортах на отопительные приборы не предоставляют весь необходимый объем информации об их технических характеристиках. А такие теплотехнические характеристики, как мощность, приводятся, в основном, для тех значений температуры теплоносителя, которые редко достигаются в большинстве систем отопления зданий. В связи с этим, точный и надежный расчет теплопотребления помещений, содержащих современные отопительные устройства, выполнить достаточно сложно.

\section{Выводы}

Как видно из представленных результатов, установка теплоотражающего экрана за радиатором снижает общие потери теплоты через внешние ограждения на 6,1 \%..6,5 \% . Но при этом снижается также температура воздуха в помещении. Для поддержания температуры воздуха на необходимом уровне необходимо повышать тепловой поток от радиатора путем увеличения температуры теплоносителя на входе в данное отопительное устройство. Это приводит к некоторому увеличению тепловых потерь через ограждения. В результате, экономия тепловой энергии от установки теплоотражающего экрана составляет $\sim 3 \%$.

Представленные результаты имеют лишь приближенный и оценочный характер. Более точные расчетные данные можно получить при решении задачи теплообмена в помещении в полной теплофизической постановке, учитывающий аэродинамику внутренних воздушных течений, локальную структуру теплопотерь через стены и окна, и ряд других важных факторов [3].

\section{ЛИТЕРАТУРА}

1. Богословский В.Н. Отопление / В.Н. Богословский, А.Н. Сканави - М: Стройиздат.- 1991.$735 \mathrm{c}$.

2. Басок Б.И. Температурный режим отапливаемого помещения. Приближенная теплофизическая модель / Б.И. Басок, Б.В. Давыденко, С.М. Гончарук, О.Н. Лысенко // Промышленная теплотехника .- 2013.- Т.35, № 4.- С. 23-30.

3. Басок Б.И. Численное моделирование температурного режима в помещении с двухпанельным радиатором / Б.И. Басок, Б.В. Давыденко, Г.Г. Фаренюк, С.М. Гончарук // Инженерно-физический журнал.-2014.-T.87, №6.- С. 1375 - 1379. 
CALCULATION THE AMOUNT OF HEAT ENERGY CONSUMPTION FOR HEATING THE ROOM BY

CAST- IRON RADIATOR M-140. PART 1. STATIONARY HEATING REGIME

Basok B.I., Davydenko B.V., Timoshchenko A.V.

Institute of Engineering Thermophysics of the National Academy of Sciences of Ukraine, vul. Zhelyabova, 2a, Kyiv-57, 03057, Ukraine

On the basis of approximate thermophysical model the temperature regime of room which is heated by cast-iron radiator is calculated. We consider the stationary heating regime, in which the heat agent temperature and the temperature of outdoor air do not change over time. The dependences of the air room temperature and of the amount of heat energy consumption on the radiator temperature are obtained. The effect of heat-reflecting screen installed on the wall behind the radiator area on heat loss is explored. It is shown that under the condition of maintaining the room temperature at $20^{\circ} \mathrm{C}$, the heat loss due to the installation of the heat-reflecting screen is reduced by $3 \%$.

References 3 , figures 2 .

Key words: heating of the room, temperature regime, radiator.

1. Bogoslovskiy V.N. Heating/V.N. Bogoslovskiy, A.N. Skanavi-M: Stroyizdat. -1991. - 735 p. (Rus.)

2. Basok B.I. Temperature regime of the heated room. An approximate thermophysical model / B.I. Basok, B.V. Davydenko, S.M. Goncharuk, O.N. Lysenko // Promyshlennaya teplotekhnika. - 2013. - V. 35, № 4, P. 23-30. (Rus.)

3. Basok B.I. Computational modeling of the temperature regime in a room with a two-panel radiator / B.I. Basok, B.V. Davydenko, G.G. Farenuyk, S.M. Goncharuk // Inzhenerno-Fizicheskii Zhurnal. - 2014. -V.87, №6. - P. 1375 - 1379. (Rus.) 